\title{
Transverse coloplasty pouch and colonic J-pouch for rectal cancer - a comparative study
}

\author{
J. M. Pimentel*, A. Duarte*, C. Gregórioł, P. Souto† and J. Patrício* \\ *Department of Surgery I, Unit of Coloproctology and †Gastroenterology Department, University Hospital of Coimbra, Coimbra, Portugal
}

Received 3 October 2002; accepted I December 2002

\begin{abstract}
Objectives The introduction of the colonic J-pouch has markedly improved the functional outcome of restorative rectal cancer surgery. However colonic J-pouch surgery can be problematic and may present some late evacuatory problems. To overcome these limitations a novel pouch has been proposed: the transverse coloplasty pouch. The purpose of our study was to compare the functional outcomes of these two different types of pouches - the transverse coloplasty pouch (TCP) and the colonic J-pouch (CJP) - during the first 12 months postoperatively.
\end{abstract}

Patients and methods A prospective randomized trial was conducted in which a total of 30 patients with mid and low rectal cancer were submitted either to a transverse coloplasty pouch or a colonic J-pouch. Clinical defaecatory function was assessed and anorectal physiological assessment was carried out, pre-operatively and at 3,6 and 12 months postoperatively, by means of a standard clinical questionnaire and by anorectal manometry.

Results No statistically significant differences were found between the two groups regarding bowel function. The postoperative frequency of daily bowel movements was lower in the TCP group in all the phases of the study
(3.9 vs. 4.1 at 3 months; 3.1 vs. 3.4 at 6 months; 2.1 vs. 2.8 at 12 months), the same occurring with fragmentation $(33 \%$ vs. $40 \%$ at 3 months; $26.6 \%$ vs. $33.3 \%$ at 6 months; $7.1 \%$ vs. $14.3 \%$ at 12 months). Less urgency was also seen in the TCP group during the first 6 months $(20 \%$ vs. $26.7 \%)$, with identical values at 12 months (14.3\% vs. $14.3 \%)$. No significant differences were also found concerning incontinence grading and scoring, with TCP patients having less nocturnal leaks. At one year two CJP patients (14.3\%) needs the use of enemas to evacuate the pouch and provoke defaecation, a problem never seen in TCP patients. The anorectal manometry data was similar in both types of pouches. The local complication rates were also identical in the two groups $(20 \%)$; more anastomotic leaks were seen in TCP patients $(13.2 \% v s$. $6.6 \%)$, without reaching a statistical significance.

Conclusion The transverse coloplasty pouch has similar functional results but fewer evacuation problems than the J-Pouch, making it a safe and reliable alternative to the colonic J-pouch.

Keywords Transverse coloplasty pouch; colonic J-pouch; rectal cancer; bowel function

\section{Introduction}

The surgical treatment of tumours of middle and distal rectum by means of a low anterior resection with a straight colorectal or coloanal anastomosis is commonly accompanied by poor bowel function. As a consequence of the loss of the rectal reservoir function, of a low neorectal capacity and of injury to the anal sphincters,

Podium presentation at the annual meeting of the European Association of Coloproctology, Erlangen, Germany, 12-14 September 2002.

Correspondence to: Professor J. M. Pimentel, Department of Surgery I, Unit of Coloproctology, University Hospital of Coimbra, Avenue. Bissaya Barreto 3000075 Coimbra, Portugal.

E-mail: cirurgial@huc.min-saude.pt patients may have an increase in defaecatory frequency, urgency and incontinence, a clinical picture that characterizes the 'anterior resection syndrome' [1-3].

To obviate these problems, Lazorthes et al. [4] and Parc et al. in 1986 [5] described the colonic-J-pouch (CJP) which, by increasing the neorectal volume, has markedly improved the functional outcome of restorative rectal cancer surgery. Several prospective randomized trials have demonstrated the functional superiority of the colonic-J-pouch over the straight colorectal or coloanal anastomosis during the first two years after surgery and even at a late time [6-9].

However, $10 \%$ to $30 \%$ of patients with the colonicJ-pouch may experience some late evacuation problems 
with incomplete defaecation that needs the daily use of laxatives, enemas and suppositories $[10,11]$. This can happen even with the construction of smaller pouches (5-6 cm), with a short limb length of 5-6 cm [12-15]. Furthermore, the construction of the colonic-J-pouch can be problematic, with technical difficulties occurring when dealing with a thick or short mesocolon or in cases of a male narrow pelvis [16].

To overcome these limitations a novel pouch has been proposed, the transverse coloplasty pouch (TCP). First described experimentally in a porcine model $[17,18]$ and subsequently in the human setting $[16,19,20]$, it is claimed that this small-volume reservoir, similar to a pyloroplasty or to a strictureplasty, gives an improvement in early functional outcomes, a decrease in late evacuation problems and some technical advantages over the colonic-J-pouch [16-22]. The only randomized trial, comparing both types of pouches, published so far indicated a higher anastomotic leak rate in the TCP group but minimal differences in bowel function between the two groups [23].

The purpose of our study was, by means of a prospective randomized trial, to compare the functional outcomes of the two different types of pouches, the transverse coloplasty pouch and the colonic-J-pouch, during the first 12 months postoperatively.

\section{Patients and methods}

From May 1999 to April 2001, 30 consecutive patients with mid or low rectal cancers (located less than $12 \mathrm{~cm}$ above anal verge) were randomized into the TCP group ( $n=15)$ or into the CJP group $(n=15)$. Functional evaluation was made, pre-operatively and at 3,6 and 12 months postoperatively (after stoma closure), by means of a standard clinical questionnaire and by anorectal manometry (Synetics 4-channel, Medtronic Functional Diagnostics, Skovlunde, Denmark). The clinical questionnaire included items concerning the frequency of bowel movements, urgency (the ability to defer defaecation for more than $15 \mathrm{~min}$ ), fragmentation (the inability to empty the pouch in one attempt and the need to defaecate again in one hour), continence and the use of laxatives, enemas or suppositories to provoke defaecation. Incontinence was recorded as grade 1 (gas), grade 2 (liquids) and grade 3 (solids), and was assessed by using a validated faecal incontinence score [24]. All patients were submitted to a routine standardized follow-up protocol, scheduled to a three months interval.

Statistical analysis was performed using the $\chi^{2}$ test and Student's $t$-test. A $P$-value of $<0.05$ was considered significant.

\section{Surgical technique}

A standardized rectal dissection was performed in both groups [25]. This included a total mesorectal excision, high ligation of the inferior mesenteric vessels, preservation of the autonomic nervous plexus and mobilization of the splenic flexure. If a double-stapled technique was used for anastomosis, a PI 30 stapler (AutoSuture Company, USSC, Norwalk, USA) was applied at or just proximal to the level of the anorectal junction; if a handsewn anastomosis was used, a mucosectomy or an intersphincteric dissection was performed and the anastomosis was done at the dentate line. The pouches were constructed with descending colon and only used if the anastomosis was done at or below $4 \mathrm{~cm}$ above the anal verge.

The transverse coloplasty pouch was performed as previously described [20]. An 8-cm longitudinal colotomy was made between the taenia along the antimesenteric border of the colon, 4-6 cm proximal to the distal cut end. The incision was closed transversely with a single layer of interrupted 2-0 polyglactin seromuscular sutures. The TCP was then anastomosed in an end-to-end fashion at the level of the anorectal junction by a double-stapled technique using a 31-mm Premium CEEA curved stapler (AutoSuture Company) or at the level of the dentate line by a handsewn technique, depending on safe distal margins.

The colonic-J-pouch was performed as previously described [4]. Two $6 \mathrm{~cm}$ colon limbs were folded, a colotomy was made at the apex of the $\mathrm{J}$, and a side-to-side anastomosis was done by introducing a linear stapler (GIA 60, AutoSuture Company) fired for $5 \mathrm{~cm}$. The pouch was then anastomosed in a side-to-end fashion at the level of the anorectal junction by a double-stapled technique using a 31-mm Premium CEEA curved stapler (AutoSuture Company) or at the level of the dentate line by a handsewn technique, depending on safe distal margins.

After completion of the anastomosis, the integrity was routinely tested with an air insufflation technique. A loop ileostomy was routinely performed and closed by 10 weeks after the initial surgical procedure. Before closure an water-soluble enema was made to exclude any leak.

\section{Results}

Both groups were well-matched for gender, mean-age, level of the tumour from anal verge, staging, neoadjuvant therapy, level of anastomosis from anal verge, type of anastomosis (stapled vs. handsewn) and use of a protective stoma. Their clinical and operative parameters are detailed in Table 1. One CJP randomized patient 
Table I Clinical and operative parameters.

\begin{tabular}{lll}
\hline & TCP & CJP \\
\hline Patients & 15 & 15 \\
Gender (male/female) & $9 / 6$ & $8 / 7$ \\
Age (mean; years) & 60.2 (range 33-83) & 62.3 (range 37-84) \\
Tumour level above anal verge & 5.1 (range 2.5-9) & 5.3 (range 2.5-10) \\
$\quad$ (mean; cm) & $4(26.7 \%)$ & $3(20 \%)$ \\
TNM I & $7(46.6 \%)$ & $7(46.6 \%)$ \\
II & $4(26.7 \%)$ & $5(33.4 \%)$ \\
III & $33.3 \%$ & $40 \%$ \\
Neo-adjuvant therapy & $2.7($ range $1.5-3.8)$ & 2.9 (range $1.5-4)$ \\
Mean level of anastomosis above anal & & \\
verge (cm) & $11 / 4$ & $12 / 3$ \\
Type of anastomosis (stapled/handsewn) & Standard TME & Standard TME \\
Operative technique & 15 & 15 \\
Protective stoma (ileostomy) & & \\
\hline
\end{tabular}

$P=$ NS (not significant). with a bulky J-pouch that would not fit into the pelvis, had a change in the surgical strategy with the construction of a TCP.

There was no 30-day postoperative mortality in either group. Two patients, one with a TCP and the other with a CJP suffered a local recurrence at 9 months and a local and systemic recurrence at 11 months, enabling just 14 patients in each group to be analysed 12 months postoperatively. Local postoperative complications directly related to the construction of the pouch occurred in the same number of patients in the TCP and CJP groups: 3 patients $(20 \%)$. More anastomotic leaks were seen in the TCP group in comparison with the CJP group (13.2\% vs. $6.6 \%$ ), a figure that did not reach a statistical significance. Clinical anastomotic leakage was identical in both groups, occurring in $1(6.6 \%)$ patient in each group, with 1 (6.6\%) further patient in the TCP group having an asymptomatic radiological leak. Anastomotic stricture requiring surgical trans-anal dilatation by a crossplasty technique were also seen in $1(6.6 \%)$ patient in each group (Table 2).

No statistical significant differences were found in bowel function between the two groups (Table 3 ). The postoperative frequency of daily bowel movements was lower in the TCP group in all the phases of the study (3.9

Table 2 Local postoperative complications.

\begin{tabular}{lll}
\hline & TCP & CJP \\
\hline Anastomotic leak & $2(13.2 \%)$ & $1(6,6 \%)$ \\
Pouch-vaginal fistula & - & $1(6.6 \%)$ \\
Anastomotic stricture & $1(6.6 \%)$ & $1(6.6 \%)$ \\
Total & $3(20 \%)$ & $3(20 \%)$ \\
\hline
\end{tabular}

$P=$ NS (not significant). vs. 4.1 at 3 months; 3.1 vs. 3.4 at 6 months; 2.1 vs. 2.8 at 12 months). The same occurred with fragmentation (33\% vs. $40 \%$ at 3 months; $26.6 \%$ vs. $33.3 \%$ at 6 months; $7.1 \%$ vs. $14.3 \%$ at 12 months). Less urgency was also seen in TCP patients during the first 6 months $(20 \%$ vs. $26.6 \%$ ); however, at 12 months the number of patients with urgency was identical in TCP and CJP groups (14.3\% vs. $14.3 \%)$. All the patients with TCP recovered daily bowel activity at 6 months, which was maintained at 12 months, a finding that was not seen in the CJP group. No significant differences were found concerning incontinence grading and scoring. The TCP patients complained of more grade 1 incontinence (gas), particularly during the first 6 months; however, they had less nocturnal leaks, a finding that at 6 months almost reach a statistical difference $(6.6 \%$ vs. $20 \%)$. At one year 2 (14.3\%) CJP patients needs the use of enemas to evacuate the pouch and provoke defaecation, a problem that began at 6 months after surgery, and was never seen in TCP patients. The percentage of patients requiring antidiarrhoeal medications was similar in the two groups.

The anorectal manometry results, pre-operatively and at 3,6 and 12 months postoperatively, showed no significant difference between the two groups. The mean resting anal pressure and the maximal anal squeeze pressure were almost similar in TCP and CJP patients, regaining the pre-operative values one year after surgery. The mean maximal tolerated volume at 3 and 12 months postoperatively was higher in the CJP patients (Table 4).

\section{Discussion}

A poor functional outcome is expected to occur after a low anterior resection with a straight coloanal anastomosis. Most patients experience an increase in stool 
Table 3 Postoperative bowel function.

\begin{tabular}{|c|c|c|c|c|c|c|}
\hline & \multicolumn{2}{|l|}{3 months } & \multicolumn{2}{|l|}{6 months } & \multicolumn{2}{|l|}{12 months } \\
\hline & ТCP & CJP & TCP & CJP & TCP & CJP \\
\hline \multicolumn{7}{|l|}{ Mean stool frequency } \\
\hline Total per $24 \mathrm{~h}$ & $3.9(1-10)$ & $4.1(1-12)$ & $3.1(1-8)$ & $3.4(1-6)$ & $2.1(1-3)$ & $2.8(1-5)$ \\
\hline Day & $2.1(1-10)$ & $3.3(1-12)$ & $2.3(1-8)$ & $2.6(1-6)$ & $1.8(0-4)$ & $2.5(0-4)$ \\
\hline Night & $1.8(1-8)$ & $0.8(0-3)$ & $0.8(0-4)$ & $0.8(0-6)$ & $0.3(0-4)$ & $0.3(0-5)$ \\
\hline Urgency & $3(20)$ & $4(26.7)$ & $3(20)$ & $4(26.7)$ & $2(14.3)$ & $2(14.3)$ \\
\hline Fragmentation & $5(33)$ & $6(40)$ & $4(26.7)$ & $5(33.3)$ & $1(7.1)$ & $2(14.3)$ \\
\hline Daily bowel activity & $14(93.3)$ & $11(73.3)$ & $15(100)$ & $12(86.7)$ & $15(100)$ & $12(86.7)$ \\
\hline Incontinence (grade) & $5(33.3)$ & $3(20)$ & $4(26.7)$ & $2(13.3)$ & $2(14.3)$ & $1(7.1)$ \\
\hline Gas & 4 & 2 & 4 & 2 & 2 & 1 \\
\hline Liquids & 1 & 1 & - & - & - & - \\
\hline Incontinence (score) & 3.2 & 2.8 & 2.9 & 2.6 & 2.7 & 2.3 \\
\hline Nocturnal leaks & $2(13.3)$ & $3(20)$ & $1(6.6)$ & $3(20)$ & $1(7.1)$ & $2(14.3)$ \\
\hline Enema use & - & - & - & $2(13.3)$ & - & $2(14.3)$ \\
\hline Anti-diarrhoeal drugs & $3(20)$ & $4(26.7)$ & $3(20)$ & $3(20)$ & $2(14.3)$ & $2(14.3)$ \\
\hline
\end{tabular}

Figures in parenthesis refer to mean stool frequency ranges and percentages in each group. $P=$ NS (not significant).

Table 4 Anorectal manometry.

\begin{tabular}{|c|c|c|c|c|c|c|c|c|}
\hline & \multicolumn{2}{|l|}{ Pre-operative } & \multicolumn{2}{|l|}{3 months } & \multicolumn{2}{|l|}{6 months } & \multicolumn{2}{|l|}{12 months } \\
\hline & ТCP & CJP & TCP & CJP & TCP & CJP & TCP & CJP \\
\hline $\begin{array}{l}\text { Rest pressure } \\
(\text { mean/mmHg) }\end{array}$ & $50.1(12.3)$ & $52.4(11.4)$ & $43.6(8.9)$ & $45.2(9.5)$ & $43.6(8.9)$ & $45.2(9.5)$ & $49.5(10)$ & $50.1(11.2)$ \\
\hline $\begin{array}{l}\text { Max pressure } \\
(\text { mean } / \mathrm{mmHg})\end{array}$ & $136.3(15.2)$ & $121.2(8.9)$ & $108.2(12.1)$ & $92.6(9.2)$ & $116.3(11.9)$ & $111.3(9.7)$ & $122.1(12.4)$ & 128.3 \\
\hline $\begin{array}{l}\text { Max tolerated volume } \\
(\mathrm{mean} / \mathrm{ml})\end{array}$ & - & - & $118.2(36.3)$ & $138.7(41.4)$ & - & - & $126.1(40.1)$ & $143.2(48.3)$ \\
\hline
\end{tabular}

Figures in parenthesis are standard error of the mean. $P=$ NS (not significant).

frequency, urgency and occasional incontinence, the so-called 'anterior resection syndrome'. To replace the rectum, the colonic-J-pouch was originally described, in $1986[4,5]$. This type of pouch has significantly improved the functional results after restorative rectal cancer surgery $[8,9,14]$ and has become a standard procedure for anastomosis at or below $4 \mathrm{~cm}$ from the anal verge [26]. However, even with small volume J-pouches of 5-6 cm, some late evacuatory problems concerning difficult pouch emptying may occur in $10 \%$ of patients [12-14]. Furthermore, the construction of the colonic-Jpouch can sometimes be problematic, not only where the apex of the pouch does not reach the proposed level of anastomosis due to a short mesocolon, but also when a pouch, due to a thick mesocolon, is too bulky to descend into a narrow pelvis [20].

The transverse coloplasty pouch was initially described experimentally in a porcine model [17] and then introduced in the clinical setting $[16,19,20]$. With a volume between a straight coloanal anastomosis and a colonic-J-pouch, it is claimed that this is an improvement with a decrease in the long-term evacuation problems of the colonic-J-pouch.

The rate of local complications $(20 \%)$ was identical in both groups. More anastomotic leaks have, however, occurred in TCP patients $(13.2 \%)$ than in CJP patients $(6.6 \%)$, but these differences were without statistical significance. In the only prospective and randomized study published so far [23], a significantly greater rate of anastomotic leak was reported in TCP patients (15.9\%) compared with none in CJP patients. In another comparative but nonrandomized study a rate of $5 \%$ clinical anastomotic leak in TCP patients compared with one minor leak in the CJP group (6.2\%) was reported [21]. In the study of Z'graggen et al. [22], the anastomotic leak rate of TCP patients was $7 \%$. 
It is apparent, from different published series $[8,14,15]$, that the leak rate in patients with a straight anastomosis is somewhat higher than after colonic pouchanal anastomosis $(5-27 \%$ vs. $0-15 \%)$. In a revision of all outstanding articles published about the colonic-J-pouch, the reduction of anastomotic leak seen with the J-pouch was attributed to a better blood flow at the site of the pouch anastomosis (side-to-end) and also to temporary faecal diversion [14]. The anastomotic leak rate seen in our TCP patients is similar to other experience [23]. The clinical TCP leaks in our study were similar to other reports [21-23], similar also to various CJP published series $[10,13]$. In the light of these findings, TCP patients are not more prone to anastomotic leakage. One patient in each group was complicated by an anastomotic stricture needing surgical dilatation. It is known that stricture is more common in patients having an anastomotic leak [8], yet no such a complication was seen in those cases.

Although some differences were seen in bowel function, these did not reach a statistical significance between the groups, each having a better functional outcome with time. Patients with TCP had slightly less stool frequency, urgency and fragmentation. There were also no significant differences between TCP and CJP patients when comparing incontinence and nocturnal leakage. However, at 6 months, TCP patients had a substantially less incidence of nocturnal leaks, almost reaching significant difference, a finding similar to others at 4 months [23]. Interestingly, in the TCP group the reduction in the frequency of bowel movements, urgency, fragmentation and incontinence occured mainly by 6 months, as already noticed by Z'graggen et al. [22]. At a similar time, function had recovered in all these patients, which did not occurr in CJP patients. It may be that TCP undergoes progressive adaptation during the first six postoperative months, with a marked benefit on bowel function after this period.

None of the TCP patients had difficulty in pouch evacuation, a problem that occurred in the CJP group. The need to use enemas was only seen in CJP patients, a limitation that began at 6 months and persisted at 12 months. Although these findings were not significant, it seems that the TCP can decrease some of the late evacuatory problems associated with the J-pouch. The need of antidiarrhoeal drugs, was no different between the two groups. At 6 and 12 months the figures were identical, with slightly more drug use by CJP patients at 3 months.

No significant difference was seen when comparing the anorectal manometry reported by others [21,23]. Both groups had similar resting and maximal squeeze pressures pre-operatively and at 3, 6, and 12 months.
A greater maximal tolerated volume was observed in J-pouches, due to a larger reservoir capacity compared with the smaller TCP [17].

The J-pouch may result in a bulky pouch that would be too large for the pelvis with a compromise in its function. With the transverse coloplasty pouch this problem does not occur suggesting an advantage for this reconstruction.

The physiological basis of the apparently better function of TCP needs to be clarified. There may be a capacitance advantage $[17,22]$, but there may also be motility factors as a consequence of the coloplasty itself $[17,23]$. Owing to the relatively small number of patients enrolled so far and the short follow-up of our study (one year), futher studies with more patients and longer follow-up are needed to confirm the results of the present study.

\section{Conclusion}

These preliminary data of our ongoing trial shows that the transverse coloplasty pouch has similar functional results but probably fewer evacuation problems than the J-pouch.

\section{References}

1 Lewis WG, Martin IG, Williamson ME et al. Why do some patients experience poor functional results after anterior resection of the rectum for carcinoma? Dis Colon Rectum 1995; 38: 259-63

2 Ortiz H, Armendariz P. Anterior resection. do the patients perceive any clinical benefit? Int J Colorectal Dis 1996; 11: 191-5.

3 Ho Y-H, Tsang C, Tang CL et al. Anal sphincter injuries from stapling instruments introduced transanally. Randomised, controlled study with endoanal ultrasound and anorectal manometry. Dis Colon Rectum 2000; 43: 169-73.

4 Lazorthes F, Fages P, Chiotasso P et al. Resection of the rectum with construction of a colonic reservoir and coloanal anastomosis for carcinoma of the rectum. Br J Surg 1986; 73: $136-8$.

5 Parc R, Tiret E, Frileux P et al. Resection and coloanal anastomosis with colonic reservoir for rectal carcinoma. $\mathrm{Br} J$ Surg 1986; 73: 139-41.

6 Lazorthes F, Chiotasso P, Gamagami RA et al. Late clinical outcome in a randomized prospective comparison of colonic J pouch and straight coloanal anastomosis. Br J Surg 1997; 84: 1449-51.

7 Seow-Choen F, Goh HS. Prospective randomised trial comparing $\mathrm{J}$ colonic pouch-anal anastomosis and straight coloanal reconstruction. Br J Surg 1995; 82: 608-10.

8 Hallböök O, Pählman L, Krog $\mathrm{M}$ et al. Randomised comparison of straight and colonic $\mathrm{J}$ pouch anastomosis after low anterior resection. Ann Surg 1996; 224: 58-65. 
9 Harris GJC, Lavery IC, Fazio YW. Function of a colonic J pouch continues to improve with time. Br J Surg 2001; 88: 1623-7.

10 Mortensen NJ, Ramirez JM, Takeuchi N et al. Colonic J pouch-anal anastomosis after rectal excision for carcinoma: functional outcome. Br J Surg 1995; 82: 611-3.

11 Berger A, Tiret E, Parc R et al. Excision of the rectum with colonic J pouch-anal anastomosis for adenocarcinoma of the low and mid rectum. World J Surg 1992; 16: 470-7.

12 Lazorthes F, Gamagami R, Chiotasso P et al. Prospective, randomised study comparing clinical results between small and large colonic J-pouch following coloanal anastomosis. Dis Colon Rectum 1997; 40: 1409-13.

13 Hida J, Yasutomi M, Maruyama T et al. Functional outcome after low anterior resection with low anastomosis for rectal cancer using the colonic J-pouch. Prospective, randomized study for determination of optimum pouch size. Dis Colon Rectum 1996; 39: 986-91.

14 Dennett ER, Parry BR. Misconceptions about the colonic J-pouch. What the accumulating data show. Dis Colon Rectum 1999; 42: 804-11.

15 Dehni N, Tiret E, Singland JD et al. Long-term functional outcome after low anterior resection. comparison of low colorectal anastomosis and colonic J-pouch-anal anastomosis. Dis Colon Rectum 1998; 41: 822-3.

16 Fazio VW. (1998) Methods to facilitate length in the colorectal anastomosis. Invited lecture at the meeting of the American Society of Colon and Rectal Surgeons, May, San Antonio, USA.

17 Z'graggen K, Maurer CA, Mettler D et al. A novel colon pouch and its comparison with a straight coloanal J-pouch anastomosis: preliminary results in pigs. Surgery 1999; 125: 105-12.

18 Maurer CA, Z'graggen K, Zimmermann W et al. Experimental study of neorectal physiology after formation of transverse coloplasty pouch. Br J Surg 1999; 86: 1451-8.

19 Z'graggen K, Maurer CA, Büchler MW. Transverse coloplasty pouch. A novel neorectal reservoir. Dig Surg 1999; 16: 363-6.

20 Fazio VW, Mantyh CR, Hull TL. Colonic «Coloplasty $\gg$. Novel technique to enhance low colorectal or coloanal anastomosis. Dis Colon Rectum 2000; 43: 1448-50.

21 Mantyh CR, Hull TL, Fazio VW. Coloplasty in low colorectal anastomosis. Manometric and functional comparison with straight and colonic J-pouch anastomosis. Dis Colon Rectum 2001; 44: 37-42.

22 Z'graggen K, Maurer CA, Birrer S et al. A new surgical concept for rectal replacement after low anterior resection. The transverse coloplasty pouch. Ann Surg 2001; 234: 780-7.

23 Ho Y-H, Brown S, Heah S-M et al. Comparison of J-pouch and coloplasty pouch for low rectal cancers. A randomized, controlled trial investigating functional results and comparative anastomotic leak rates. Ann Surg 2002; 236: 49-55.

24 Jorge JMN, Wexner SD. Etiology and management of faecal incontinence. Dis Colon Rectum 1993; 36: 77-97.

25 Heald RJ, Ryall RD. Recurrence and survival after total mesorectal excision for rectal cancer. Lancet 1986; 1: 147982.

26 Hida J, Yasutomi M, Fujimoto $\mathrm{K}$ et al. Indications for colonic J-pouch reconstruction after anterior resection for rectal cancer. determining the optimum level of anastomosis. Dis Colon Rectum 1998; 41: 558-63. 
Copyright $\odot 2003$ EBSCO Publishing 